\title{
RESEARCH
}

\section{Uncertainty Analysis of Quasi-Zero Stiffness Air Suspension based on Polynomial Chaos Method}

\author{
Xing $\mathrm{Xu}^{*}$, Huan Liu, Xinwei Jiang and Akolbire Vincent Atindana
}

\begin{abstract}
In order to improve the vibration isolation performance of suspensions, various new structural forms of suspensions have been proposed. In this paper, a quasi-zero stiffness isolator is used in automotive suspensions to form a new suspension-quasi-zero stiffness air suspension(QZSAS).Due to the strong nonlinearity and structural complexity of quasi-zero stiffness suspensions, changes in structural parameters may cause dramatic changes in suspension performance, so it is of practical importance to study the effect of structural parameter uncertainty on the performance of such suspensions. In order to solve this problem, three suspension structural parameters $d_{0}, L_{0}, P_{c 0}$ are selected as random variables in this paper, and the polynomial chaos expansion(PCE) theory is used to solve the suspension performance parameters such as body acceleration, suspension dynamic deflection, and wheel relative dynamic load response under different excitation. The sensitivity of the performance parameters to different structural parameters was discussed and analysed in the frequency domain. This paper verifies the feasibility of the PCE method for solving the uncertainty problem of complex nonlinear systems, and the research in this paper can provide a reference for the future structural design and optimization of such suspension systems.
\end{abstract}

Keywords: Quasi-Zero Stiffness; Polynomial Chaos; Automobile Suspension

\footnotetext{
${ }^{*}$ Correspondence: xuxing@mail.ujs.edu.cn

Automotive Engineering Research Institute, Jiangsu University, Zhenjiang, China

Full list of author information is available at the end of the article
}

\section{Introduction}

The suspension system is an essential part of automobiles, which affects ride comfort, handling stability and driving safety. In order to improve the suspension performance, various new structures for suspensions have been proposed. The suspension serves as a vibration isolation system, and much of its structural design depends on the type of vibration isolator. A quasi-zero stiffness isolator is a nonlinear vibration isolation system with high static stiffness and low dynamic stiffness(Palomares et al. (2018)). Alabuzhev and Gritchin (1989) first put forward the concept of quasi-zero stiffness, using the parallel principle of positive and negative stiffness, a variety of quasi-zero stiffness structures were designed and their mechanical properties were analyzed. Through theoretical and experimental analysis, Le and Ahn (2013) proved that the system has a larger vibration isolation frequency range and higher vibration attenuation rate compared with the system without negative stiffness. Although the quasi-zero stiffness isolator has attracted much attention from scholars because of its excellent vibration isolation performance, few scholars have applied it to suspensions and studied the influence of structural parameters uncertainties on its performance.

The parameters in a vehicle are generally considered as deterministic, but there are still many uncertain parameters, which can be made by design tolerance, manufacture error, and/or time-varying, which greatly affects vehicle dynamic performance. Thus it is very important to consider the uncertainty of parameters in the analysis of suspension performance(Feng et al. (2013)). Nagy and Braatz (2010) classified uncertainty propagation methods available into three categories: (i) analytical methods, (ii) Monte Carlo simulation methods, and (iii) response surface methods, and stated that analytical methods are the most efficient but can be applied for a very limited class of systems represented by simple models. The most accurate of these is Monte Carlo(MC) simulation, but this often requires thousands of deterministic simulations to obtain uncertainty statistics, which is infeasible for computationally expensive CFD simulations(Dodson and Parks (2015)). Dai et al. (2011) applied MC method 
to the field of automobile suspension and considered the sprung mass, unsprung mass, suspension damping, and tire stiffness as random variables. The mean value, standard deviation and variation coefficient of the vehicle's natural frequencies and mode shapes are obtained by using the MC method. Chen et al. (2017) used MC simulation results to verify the correctness of the application of Unascertained Theory in automobile suspension.

An alternative approach, which has been intensively applied in the past decades, is based on stochastic response surface (SRS), which describe the performance function/model output as a sum of elementary functions of stochastic input parameters. The model has convergent expansions similar to the power series. Polynomial chaos expansion (PCE) as a functional approximation of the mathematical model belongs to the class of SRS approaches. PCE method was first proposed by Wiener, mainly used to establish turbulence model(Wiener (1938)). Now, PCE method has been widely used in many engineering fields, such as fluid mechanics(Dodson and Parks (2015); Li et al. (2009); Xiu and Karniadakis (2003a)), Multibody dynamics(Sandu et al. (2006)), Transient heat transfer(Xiu and Karniadakis (2003b)). As a new stochastic method to solve uncertain problems. The comparison between MC method and PCE method in solving uncertain problems has also been studied by many scholars. Li et al. (2009)'s research show that the PCE method can accurately estimate the flow statistics with a much smaller computational effort than the MC method. Dodson and Parks (2015) found that a significantly lower computational burden characterizes the polynomial chaos expansion-based approach than MC approach in their research. Other researchers (Kewlani et al. (2012); Qiu et al. (2019); Xiu and Karniadakis (2002)) also came to the same conclusion.

Although many scholars have studied the uncertainty problem, the application of new structures in automotive suspensions is still relatively rare. More often, it is applied to simple linear spring-damping systems. In this paper, the quasi-zero stiffness air suspension(QZSAS), a new type of automotive suspension with quasi-zero stiffness characteristics, is proposed. The effect of structural parameters uncertainties on the suspension performance is further investigated by the PCE method. Because of the strong nonlinearity object studyed, this reseach fills the gap in the study of uncertainty of complex structural suspensions. Since the PCE method has a small computational volume and high accuracy, this paper uses the PCE method to establish the QZSAS uncertainty model, with structural parameters as uncertainty inputs and suspension performance parameters as uncertainty outputs. The article discusses the degree of influence of different structural parameter uncertainties on each suspension performance parameter. The feasibility of the PCE method and the different sensitivity to the suspensions parameters are also concluded.

\section{Quasi-Zero Stiffness Air Suspensions System}

The QZSAS studied in this paper is a suspension for commercial vehicles. The mechanism is supported by an air spring in the vertical direction to provide positive stiffness, and two opposed cylinders are placed horizontally to provide negative stiffness to achieve an overall zero stiffness.

As shown in Figure 1, there is a vertically placed air spring and two horizontally opposed cylinders installed between the vehicle body and the frame. The two ends of the cylinders are connected to the body and the frame respectively by hinges. The chamber of the cylinder near the air spring is connected to the atmosphere, and the chamber at the far end is connected to the air tank. The air in the cylinders and air spring is supplied by the high-pressure air tank, and the discharged air goes to the low-pressure air tank. Solenoid valves are installed between the air tank, the air spring and the cylinder to control inflation and deflation. The air entering the low pressure air tank can be pumped into the high pressure air tank by an air pump. As with conventional suspensions, shock absorbers are installed between the body and the frame, and the frame is connected to the wheels (not shown in Figure 1).

When the vehicle is driven on a bumpy road, the body and frame will be displaced relatively to each other, the air spring will be stretched or compressed, and the cylinder will rotate. The air chamber of the cylinder away from the air spring is filled with high pressure air (higher pressure than the atmosphere). The chamber close to the air spring is connected to the atmosphere. So the force of the cylinder on the two articulation points is always a thrust. When the relative displacement between the body and the frame increases, as shown in Figure 2(a), the air spring will be stretched, the left cylinder will rotate counterclockwise, and the right cylinder will rotate clockwise. The combined force of the frame to the body is vertical upward. When the relative displacement between the body and the frame decreases, as shown in Figure 2(b), the air spring will be compressed, the left cylinder will rotate clockwise, and the right cylinder will rotate counterclockwise. The combined force of the frame to the body is vertical downward.

Since the quasi-zero stiffness air suspension uses the compressibility of air to produce vibration isolation. The relationship between air pressure and volume in 
air springs and cylinders will be given by the gas state equation in Equation (1), where the gas mass is considered to be constant.

$$
P V^{n}=P_{0} V_{0}^{n}
$$

where $P_{0}$ is the initial state air pressure, $V_{0}$ is the initial state volume, $P$ is the air pressure in any state, $V$ is the volume in any state, and $n$ is the polytropic index.

The air spring selected is a membrane air spring. In its working air pressure range, its effective area is almost unchanged. So it will be simplified to a cylinder with the same piston area. For a certain displacement $z$, according to the gas state Equation (1), the size of its force as shown in Equation (2).

$$
F_{s}=A_{s}\left[P_{s 0}\left(\frac{z_{0}}{z_{0}-z}\right)^{n}-P_{a}\right]
$$

Where $A_{s}$ is the effective area of the air spring, $P_{s 0}$ is the air spring pressure in the equilibrium position, $z_{0}$ is the height of the air spring in the equilibrium position, $P_{a}$ is the atmospheric pressure.

Figure 3 shows the movement of left cylinder when the vehicle driving on the bumpy road.$d_{0}$ is the distance from the piston to the back wall of the highpressure chamber and $L_{0}$ is the distance between the two hinge points of the cylinder when the vehicle is stationary. When the body and the frame have relative displacement, the cylinder will rotate. $\theta$ is the cylinder rotation angle, $z$ is the displacement of the body relative to the frame, $d$ is the distance from the piston to the back wall of the high-pressure chamber and $L$ is the distance between the two hinge points of the cylinder when the vehicle is in motion. The geometric relationship of these parameters can be given by Equation (3) (4) (5).

$$
\begin{array}{r}
L=\sqrt{L_{0}^{2}+z^{2}} \\
\sin \theta=\frac{z}{\sqrt{L_{0}^{2}+z^{2}}} \\
\Delta d=\sqrt{\left(L_{0}^{2}+z^{2}\right)}-L_{0}
\end{array}
$$

Then through force analysis, negative stiffness cylinder in the vertical direction of the force can be given by the Equation (6).

$$
F_{c}=2 A_{c}\left[P_{c 0}\left(\frac{d_{0}}{d_{0}+\Delta d}\right)^{n_{c}}-P_{a}\right] \sin \theta
$$

where $A_{c}$ is the effective area of the cylinder, $P_{c 0}$ is the cylinder equilibrium position air pressure, and $n_{c}$ is the gas polytropic index inside the cylinder.

$$
\left\{\begin{array}{l}
m_{1} \ddot{z}_{1}+C\left(\dot{z_{1}}-\dot{z_{2}}\right)-F=0 \\
m_{2} \ddot{z}_{2}-C\left(\dot{z_{1}}-\dot{z_{2}}\right)+K_{t}\left(z_{2}-q\right)+F=0
\end{array}\right.
$$

After determining the forces provided by the positive and negative stiffnesses, according to the quasi-zero stiffness 1/4 air suspension model shown in Figure 2, the dynamics equations of the system can be obtained, given by Equation (7). Where $C$ is the damping coefficient and $K_{t}$ is the tire stiffness. the value of $\mathrm{F}$ can be obtained from Equation (8).

$$
F=F_{s}\left(z_{1}-z_{2}\right)+F_{c}\left(z_{1}-z_{2}\right)-m_{1} g
$$

Where $F_{s}\left(z_{1}-z_{2}\right)$ is meant to be a function of the distance $\left(z_{1}-z_{2}\right)$ between the body and frame, and similarly for $F_{c}\left(z_{1}-z_{2}\right)$.These two can be calculated separately by Equation (2) and (6).

\section{Polynomial Chaos Expansion Method}

The PCE method is actually an alternative model approach. For a complex model of a sufficiently smooth black-box system in probability space, as represented by Equation (9).

$$
Y=f(X)
$$

$X$ is the input to the black box and $Y$ is the output of the black box, but the correspondence is not known. Usually, black box systems are highly nonlinear and difficult to express in explicit terms. The essence of polynomial chaos expansion is to approximate this black box by a set of polynomials. This alternative model can be expressed as Equation (10).

$$
\begin{aligned}
& Y=c_{0}+\sum_{i_{1}=1}^{n} c_{i_{1}} \Gamma_{1}\left(\xi_{i_{1}}\right)+\sum_{i_{1}=1}^{n} \sum_{i_{2}=1}^{i_{1}} c_{i_{1} i_{2}} \Gamma_{2}\left(\xi_{i_{1}}, \xi_{i_{2}}\right) \\
& +\sum_{i_{1}=1}^{n} \sum_{i_{2}=1}^{i_{1}} \sum_{i_{3}=1}^{i_{2}} c_{i_{1} i_{2} i_{3}} \Gamma_{3}\left(\xi_{i_{1}}, \xi_{i_{2}}, \xi_{i_{3}}\right) \cdots
\end{aligned}
$$

where $c_{i}$ is the coefficient to be solved; $\xi_{i}$ is the uncertainty variable obeying the standard normal distribution $; \Gamma_{i}(\xi)$ is Hermite orthogonal polynomial, whose general formula can be expressed as Equation (11). 


$$
\Gamma_{\text {dim }}(\xi)=(-1)^{\operatorname{dim}} e^{\frac{1}{2} \xi^{T} \xi} \frac{\partial^{\operatorname{dim}}}{\partial \xi_{i_{1}}, \cdots, \partial \xi_{i_{d i m}}} e^{-\frac{1}{2} \xi^{T} \xi}
$$

where dim is the number of random variable dimensions. And the $\xi=\left[\xi_{i_{1}}, \xi_{i_{2}}, \cdots, \xi_{i_{\text {dim }}}\right]$.

In practical applications, it is not possible to expand the polynomial by an infinite number of terms. Usually the s-term truncation is taken to approximate the system output, and the above equation can be approximated in the form of Equation (12).

$$
Y \approx \sum_{i=0}^{s-1} c_{i} \Gamma_{i}(\xi)
$$

The recursive formula will be used instead of the defining equation of Equation (11), which facilitates the procedural implementation of the chaotic polynomial, and the recursive formula is shown in Equation (13). Here $i$ is the order of the hermite polynomial.

For computational convenience, the Hermite polynomials are normalized by dividing each term by the square root of the factorial of the order. The Hermite polynomials after normalization of the first 6 orders are given by Table 1 .

$$
\left\{\begin{array}{l}
\Gamma_{1}(\xi)=1 \\
\Gamma_{2}(\xi)=\xi \\
\Gamma_{i}(\xi)=\xi \Gamma_{i-1}(\xi)-(i-2) \Gamma_{i-2}(\xi)(i=3,4, \cdots)
\end{array}\right.
$$

The solution of the polynomial chaos coefficients $c_{i}$ is a crucial step. In general, the solution of the coefficients can be classified as intrusive or non-intrusive. For the black-box problem, it is not easy to use the invasive solution because the specific expression of the initial model cannot be determined, so the noninvasive method is chosen to calculate the coefficients in this paper. There are two commonly used methods for non-invasive solutions, the Galerkin projection method and the collection method. The collection method is easier to implement than the Galerkin method, especially for nonlinear systems(Xiu (2007)), and this paper used the collection method for solving the polynomial chaotic coefficients.

The key step is to select and combine the collocation points. For the random variables obeying Gaussian stochastic process, the expansion base should be chosen as Hermite orthogonal polynomial. Assuming that the order of expansion is $m$, then the collocation points can be chosen as the roots of $(m+1)$ th Hermite orthogonal polynomials(Isukapalli (1999)).The collocation points cannot be directly input to the system, therefore, they need to be transformed into input variables. Taking a random variable obeying Gaussian distribution as an example, the input variable $x=\sigma \xi+\mu$, where $\mu, \sigma$ are the prior mean and standard deviation of the input random variables. The process of calculating the output response statistics using the PCE method is shown in Figure 5.

If only collocation points obtained by finding the roots of the polynomial are selected for the calculation, it may lead to inaccurate calculation of the coefficients. In order to overcome this phenomenon and minimize the influence of each group of collocation points on the response surface, more collocation points can be introduced to solve the coefficients. The number of groups of collocation points is only needed to be more than the total number of coefficients to be solved, and the polynomial coefficients can be obtained by solving the system of linear equations shown in Equation (14).

$$
\left[\begin{array}{cccc}
\Gamma_{0}\left(\xi_{0}\right) & \Gamma_{1}\left(\xi_{0}\right) & \cdots & \Gamma_{s-1}\left(\xi_{0}\right) \\
\Gamma_{0}\left(\xi_{1}\right) & \Gamma_{1}\left(\xi_{1}\right) & \cdots & \Gamma_{s-1}\left(\xi_{1}\right) \\
\vdots & \vdots & & \vdots \\
\Gamma_{0}\left(\xi_{N}\right) & \Gamma_{1}\left(\xi_{N}\right) & \cdots & \Gamma_{s-1}\left(\xi_{N}\right)
\end{array}\right]\left[\begin{array}{c}
c_{0} \\
c_{1} \\
\vdots \\
c_{s-1}
\end{array}\right]=\left[\begin{array}{c}
Y\left(\xi_{0}\right) \\
Y\left(\xi_{1}\right) \\
\vdots \\
Y\left(\xi_{N}\right)
\end{array}\right]
$$

Where $N$ is the total number of sampling points, and $s$ is the number of coefficients to be solved.

Since the orthogonal polynomial has the property of weighted orthogonality, its mean and variance can be found more quickly from the coefficients of the chaotic polynomial. Equation (15) is the mean solution of the system response $Y$. Equation (16) is the variance solution of the system response $Y$.The first coefficient of the polynomial is the mean of the response, and the sum of the squares of the coefficients from the second term to the last term is the variance of the response. After the PCE model is obtained, the statistical distribution of the response can be found by bringing in the alternative model using MC method for sampling.

$$
\begin{array}{r}
\mu_{Y}=\int_{\Omega} g(\xi) f(\xi) d \xi=c_{0} \\
\sigma_{Y}^{2}=\int_{\Omega} g^{2}(\xi) f(\xi) d \xi-\mu_{Y}^{2}=\sum_{i=1}^{s-1} c_{i}^{2}
\end{array}
$$

Where $g(\xi)$ is a function of the polynomial alternative model and $f(\xi)$ is the power function of the polynomial expansion. 


\section{Using PCE on the QZSAS}

The QZSAS in this paper is a strongly nonlinear system whose mathematical model has been presented in previous section. The parameters of the suspension are given in Table 2, and the MATLAB/Simulink simulation model of the suspension is established through the data in this table.

There are many parameters of this system that can affect the overall performance of the suspension. The following parameters are selected as random variables in this paper. Their magnitudes are taken into account due to manufacturing and installation errors, sensor accuracy, equipment errors and other uncertainties.

Since the QZSAS has multiple components, errors are inevitable in the manufacturing and installation process. $d_{0}, L_{0}$ as non-manufacturing quantities, whose magnitudes are determined by other manufacturing quantities, have a wide range of variation due to installation and manufacturing errors, so they were selected as uncertain parameters in this study.

$P_{c 0}$ is the air pressure of the cylinder at the balance position of the system. Its value is determined by the delayed effect of the gas during inflation,the closing accuracy of the solenoid valve, and the accuracy of the air pressure sensor. So its actual value is inconsistent with the expected size during inflation, and there is a fluctuation range, so $P_{c 0}$ was chosen as a parameter of uncertainty study.

$$
\begin{aligned}
a, f_{d}, \frac{F_{d}}{G} & \approx \sum_{i=0}^{s-1} c_{p i} \Gamma_{i}\left(P_{c 0}\right) \\
a, f_{d}, \frac{F_{d}}{G} & \approx \sum_{i=0}^{s-1} c_{d i} \Gamma_{i}\left(d_{0}\right) \\
a, f_{d}, \frac{F_{d}}{G} & \approx \sum_{i=0}^{s-1} c_{l i} \Gamma_{i}\left(L_{0}\right)
\end{aligned}
$$

The above parameters are affected by many mutually independent random factors, and the impact of each factor is very small. So these parameters can be seen as obeying a normal distribution, the mean value of $d_{0}, L_{0}, P_{c 0}$ is in the Table 2 . If the coefficient of variation $\zeta$ is taken as $10 \%$, their probability density distribution can be given by Figure 6 .

After determining the uncertain parameters, the QZSAS is regarded as a nonlinear black box system, and the Matlab/Simulink model is used instead in this paper.The model can be built from the differential Equation (7).

The uncertain parameters matching with the standard normal distribution are brought into the simulation model. To determine the effect of parameters uncertainties on suspension performance, the body acceleration $a$, suspension dynamic deflection $f_{d}$, and wheel relative dynamic load $F_{d} / G$ used to evaluate the suspension performance are obtained. The body acceleration $a$ is the main indicator to evaluate the smoothness of the car. The dynamic suspension deflection,which is defined as the relative displacement of the body and the frame, is related to its limit travel. And its improper fit increases the probability of hitting the limit and makes the smoothness worse. The relative dynamic load of the suspension is the ratio of wheel dynamic Load $F_{d}$ between static load $G$. Where dynamic load $F_{d}$ equals to $K_{t}\left(z_{2}-q\right)$, static load $G$ equals to $\left(m_{1}+m_{2}\right) g$. The relative dynamic load affects the adhesion effect between the wheel and the road, and is related to driving safety. The expression of polynomial chaos is obtained, as given in Equation (17) (18)(19).

The coefficients of polynomial chaos can be calculated by Equation (14), and the mean and variance of $a, F_{d} / G$, and $f_{d}$ can be quickly calculated by Equation (15) (16). If further distribution of the output response is desired, the sampling points can be brought into PCE with the help of the MC method.

\section{Result and Discussion}

A total of three road excitations are selected in this paper: sine excitation, step excitation, and full frequency band excitation. And the statistical index of the output response under each excitation is derived.

The excitation chosen for the QZSAS simulation model is a sinusoidal excitation of $1 \mathrm{~Hz}$ with an amplitude of $0.1 \mathrm{~m}$. The variation coefficient of the input parameters is $10 \%$. Figure 7 shows the mean and variance plots of the acceleration response to the suspension for different random parameter inputs.

It can be seen that in comparison, the uncertainty of $P_{c 0}$ has the largest effect on the acceleration standard deviation response, and the effects of $d_{0}$ and $L_{0}$ on the standard deviation are very close. The effects of the three parameters on the mean acceleration response are almost the same. The acceleration distribution at the top is more dispersed compared to that at the bottom.

Figure 8 shows each of the three random variables on the dynamic deflection response of the suspension. $P_{c 0}$ is still the random variable that affects the standard deviation the most, and the other two random variables do not have very different effects on the standard deviation. The mean values of the deflection responses of the three random variables are almost the same.

The effects of $P_{c 0}, L_{0}, d_{0}$ on the dynamic load response of the suspension are given by Figure 9 . The effects of the three random variables on the mean and 
variance of the dynamic load response are almost the same as the acceleration and dynamic deflection.

In order to verify the correctness of the PCE method, a stochastic simulation was carried out using the $\mathrm{MC}$ method and compared with the PCE-MC method. The PCE-MC method means that the sampling points are brought into the PCE alternative model rather than into the actual model as in the MC method. The size of the random sampling points selected was 500, and the probability density profile of Figure 10 was obtained after the numerical simulation of the two methods. Where Figure 10(a),10(b)and10(c), show the probability density functions of $a, f_{d}$, and $F_{d} / G$.

From the Figure 6, it can be seen that the input random variables $d_{0}, L_{0}, P_{c 0}$ are obeying a symmetric normal distribution. As a comparison, from the Figure 10, the distributions of the output random variables $a, f_{d}$ and $F_{d} / G$ are not symmetrical but skewed distribution.

The curves of the distribution function of the output random variables calculated using the MC method and the PCE-MC method are almost the same. And the MC method is an exact verified random method. It also verifies the feasibility and validity of the PCE-MC method.

In order to compare the effects of input random variables with different variation coefficients on the response of system, two different coefficients of variation $(10 \%, 20 \%)$ are selected in this paper. The mean and variance of the response of the three random variables on the output of the system are obtained by the PCE method under the excitation of sinusoidal and step signals, respectively.

Figure 11 shows the effect of random variables with different coefficients of variation on the $a, f_{d}$, and $F_{d} / G$ responses. Also a response of the deterministic input as a comparison. The variation coefficients of the random input parameters are chosen to be $10 \%$ and $20 \%$. Figure 11(a) shows the random response curves of body acceleration. It can be seen that when the variation coefficients of the random variable is 0.1 , the effect differences of $d_{0}$ and $L_{0}$ on the mean value of the body acceleration responses is not significant, and the effect of $P_{c 0}$ is greater than that of other random variables, and the highest point of the curve is the highest point of the $\zeta=0$ curve. That means the output response of the random variable mean is not equal to the mean of the output response. The mean response curve of the suspension dynamic deflection is given by Figure 11(b), which shows that $P_{c 0}$ is still the most significant random variable affecting the mean value of the system output. $20 \%$ variation coefficient of $P_{c 0}$ is the smallest amplitude among all the curves. The other curves have more similar characteristics to Figure 11(a). The mean of $F_{d} / G$ response curves are shown in Figure $11(\mathrm{c})$, and the same by Figure $11(\mathrm{a}) 11(\mathrm{~b})$ is that $P_{c 0}$ with $20 \%$ coefficient of variation is the smallest amplitude among all the curves. This also illustrates that the output response of the system analyzed with the design value as input can show a large deviation due to the uncertainty of the parameters. Different random variables can have different effects on the system response.

To further quantify the random variables, the response mean maximum (RMM) and response variance mean (RVM) are introduced in this paper as parameters for evaluating the effect of input random variables on the output response under sinusoidal road excitation. The RMM is calculated by finding the maximum value of the output response mean, and the RVM is obtained by averaging the response variance at each simulated output point. The magnitudes of RMM and RVM for body acceleration with different input random variables at different variation coefficients $\zeta$ are given in Table 3 . And the RMM equals to $23.9361 \mathrm{~m} / \mathrm{s}^{2}$ when the body acceleration under deterministic input. By comparison, it is found that the response mean RMM of acceleration under random input is smaller than that under deterministic input. The effects of the random variable $d_{0}$ and $L_{0}$ on the random variable acceleration $a$ are closer under the same coefficient of variation. However, the effect of $L_{0}$ is smaller than that of $d_{0}$, and the variance of the output response is also the smallest among the three. The output response is most sensitive to $P_{c 0}$ under the same variation coefficient . The body acceleration response due to $P_{c 0}$ as a random variable input changes the most with respect to the deterministic input for both RMM and RVM. The corresponding RMM and RVM changed when the variation coefficient increased, and the RVM increased more significantly. Accordingly, the output response was more dispersed, but the RMM showed a slight decrease. Similarly, the RMM and RVM of the suspension dynamic deflection are also calculated in this paper. The value of its RMM is $0.3754 \mathrm{~m}$ under the deterministic input, as shown in Table 4. All three random variable inputs have a small effect on the RMM of the output response. As the same as the body acceleration, $P_{c 0}$ as the random variable input causes the largest change in the response and generate the more dispersed output relative to the other two variables. The change in the variation coefficient $\zeta$ from $10 \%$ to $20 \%$ causes a $4-6$ times change in the RVM, and it can be seen that the variation coefficient $\zeta$ can have a greater effect on the dispersion of the output response. Table 5 gives the response of the last output response, wheel dynamic load, for different random input variables, and it can be seen that the effect on the output 
is $P_{c 0}>d_{0}>L_{0}$. Similarly, the increase of $\zeta$ causes the output variable distribution to be more dispersed.

It is essential for a vehicle to pass a speed bump when driving on a city road, or suddenly a tire presses over a stone on a country road. In these cases, the performance of the vehicle suspension directly affects the smoothness and safety of the vehicle. So this paper also studies the influence of the parameters uncertainties on the vehicle suspension performance under the step road excitation, Figure 12 shows the curves of the vehicle body acceleration, suspension dynamic deflection, and wheel dynamic load response with time when the road surface is a step signal. It can be seen from Figure 12(a) that the effect of different inputs on the body acceleration is not significant enough, but there is still a small difference. The body acceleration can recover to zero value faster when the coefficient of variation of $P_{c 0}$ is $20 \%$. From the Figure $12(\mathrm{~b})$, the mean suspension dynamic deflection output is more sensitive to the difference of the variation coefficient and parameters. There is an anomaly in the figure, that is, $P_{c 0}$ and $L_{0}$ are always in the position of greater than zero after $2.5 \mathrm{~s}$ when the variation coefficient $\zeta$ is $20 \%$, which means that the deflection can not recover to the original equilibrium position. And Figure 12(c) shows that the wheel ralative dynamic load $F_{d} / G$ is more insensitive to the difference between the random variables and the coefficient of variation relative to the other two performance parameters.

For a random road surface which the vehicle travels normally, the road velocity spectrum $\sqrt{G_{\dot{q}}(\omega)}$ is white noise, and the root-mean-square value spectrum of the responses such as body acceleration, suspension dynamic deflection, and wheel relative dynamic load is the amplitude-frequency characteristic $\left|\frac{\ddot{z}}{\dot{q}}\right|,\left|\frac{f_{d}}{\dot{q}}\right|,\left|\frac{F_{d}}{G \dot{q}}\right|$ multiplied by the constant $\sqrt{G_{\dot{q}}(\omega)}$. So that one can use the amplitude-frequency characteristics of the response volume to the velocity input to qualitatively analyze the root-mean-square value spectrum of the response.

Figures 13,14,15 show the upper and lower bound curves of the amplitude-frequency characteristics of the response volume to velocity input obtained by using the PCE method. The variation coefficient of the random variables are all taken as $10 \%$, and the magnitude of the upper and lower bound curves is obtained by adding or subtracting twice the standard deviation from the derived mean value.

From Figure 13(a), it can be concluded that at frequencies less than $1 \mathrm{~Hz}$, the maximum and minimum bounds almost coincide. In the low frequency band, the uncertainty of $d_{0}$ has almost no effect on the magnitude-frequency characteristics. When the frequency is in the range of $1-4 \mathrm{~Hz}$, the maximum and minimum bound curves are not in coincidence, and most of the $|\ddot{z}|$ magnitudes fluctuate within the maximum and minimum bounds due to the uncertainty of $d_{0}$. When the frequency is greater than 4 $\mathrm{Hz}$, the two curves overlap and the effect of $d_{0}$ uncertainty on the amplitude-frequency characteristics becomes minor. As a comparison, Figure 14(a) shows the amplitude-frequency characteristic curves of $L_{0}$ as an input random variable. Unlike Figure 13(a), the difference between the maximum and minimum bounds is larger in the low frequency band. The uncertainty of $L_{0}$ in the low frequency region has a greater effect on the acceleration compared to $d_{0}$. And the range of amplitude frequency response caused by $L_{0}$ in the middle frequency band of $1-10 \mathrm{~Hz}$ is smaller than the range caused by $d_{0}$ in Figure 13(a), which indicates that the body acceleration response is more sensitive to $d_{0}$ than $L_{0}$ in the middle frequency band. When the frequency is greater than $10 \mathrm{~Hz}$, there is almost no difference between the curves of the two plots. The effect of $P_{c 0}$ on the responseis given by Figure 15(a). Comparing the other two plots, the difference between the maximum and minimum boundary curves is the largest in both the low-frequency and the mid-frequency regions. It can be concluded that the uncertainty of $P_{c 0}$ has the largest effect on the body acceleration among the three random variables. In the high frequency region, there is almost no difference between the effects of these three random variables.

Figures 13(b),14(b), and 15(b) give the effects of three random variables on suspension dynamic deflection, respectively. The influence region caused by $d_{0}$ uncertainty is mainly between 0.3 and $2 \mathrm{~Hz}$, the influence region of $P_{c 0}$ is within $1 \mathrm{~Hz}$, and $P_{c 0}$ has a large influence on suspension dynamic deflection up to $2 \mathrm{~Hz}$, but the influence is more significant in the low frequency region. By comparison, it is found that in the low frequency region with frequency less than $0.8 \mathrm{HZ}$, the influence level of uncertainty arranges as $P_{c 0}>L_{0}>d_{0}$, and in the middle frequency 0.8 $3 \mathrm{HZ}$, the influence level of uncertainty arranges as $P_{c 0}>d_{0}>L_{0}$. At other frequency band,the three random variables have the same influence level.

For the dynamic wheel load, it can be seen from Figure 13(c), 14(c), and 15(c) that the influence region caused by $d_{0}$ uncertainty is mainly between $1-10 \mathrm{~Hz}$, the influence region of $P_{c 0}$ is within $4 \mathrm{~Hz}$, and $P_{c 0}$ has a greater influence on the dynamic suspension deflection up to $10 \mathrm{~Hz}$, but the influence is more significant in the intermediate frequency region. By comparison, it is found that in the low frequency region with frequency less than $1 \mathrm{HZ}$, the influence level of uncertainty arranges as $P_{c 0}>L_{0}>d_{0}$, and in the intermediate 
frequency 1-5 HZ, the influence level of uncertainty arranges as $P_{c 0}>d_{0}>L_{0}$. The three random variables have close influence level at higher frequency band.

\section{Conclusion}

In this paper, The PCE method is applied to the QZSAS to analyze the effects of parameters uncertainties on suspension performance. The uncertainty parameters chosen in this paper are structural parameters of the suspension. The output responses were selected suspension performance parameters. The following conclusions are obtained from the simulation analysis.

(1) By comparing with the MC method, it is found that the PCE method is feasible in solving the uncertainty problem and can produce results very close to those of the MC method.

(2) Although the input variables follow a normal distribution, the distribution of the output responses is not a symmetrically but a skewed distribution.

(3) The output response distributions of the suspension is related to input random variables, the variation coefficient of the inputs, and road excitation. The sensitivity of the suspension performance parameters to different frequency road surfaces is different. The influence level of each structural parameter is focused on a certain frequency band.

The research in this paper provides a theoretical basis for the design and optimization of suspensions. It is of practical importance to consider the uncertainties of parameters when designing a suspension. The mean value of the output performance parameters reflects the overall effect of the suspension design, and the standard deviation reflects the ability of the suspension to maintain performance. In the process of design and optimization, both mean and standard deviation must be considered for the superior performance of the suspension. Future work can focus on the design and optimization of structural parameters for suspensions considering uncertainties.

\section{Availability of data and materials}

The data that support the findings of this study are openly available in "Harvard Dataverse" at https://doi.org/10.7910/DVN/YOORSR, Liu (2021).

\section{Competing interests}

The authors declare that they have no competing interests.

\section{Funding}

This work was supported by the National Natural Science Foundation of China under Grant No. 51875256.

\section{Authors' contributions}

Xing $\mathrm{Xu}$ proposed the structure of the quasi-zero stiffness air suspension also he pointed out the direction of the research and established the main structure of the paper. Huan Liu and Xinwei Jiang were responsible for programming the polynomial chaos method and building the model, and Vincent Akolbire Atindana was responsible for checking the self-consistency of the logic and the correctness of the English grammar in the paper.

\section{Acknowledgements}

Not applicable.

Author details

Automotive Engineering Research Institute, Jiangsu University, Zhenjiang, China.

\section{References}

Alabuzhev, P. M. and Gritchin, A. (1989). Vibration protecting and measuring systems with quasi-zero stiffness. Hemisphere Publishing Co. , Taylor \& Francis Group, New York.

Chen, K., Yu, X., Zheng, H., and Wang, Y. (2017). Study on the unascertained-dynamic model and dynamic response of vehicle suspension. Yingyong Lixue Xuebao/Chinese Journal of Applied Mechanics, 34:647-653.

Dai, J., Wei, G., and Zhang, N. (2011). Random displacement and acceleration responses of vehicles with uncertainty. Journal of Mechanical Science \& Technology, 25(5):1221-1229.

Dodson, M. and Parks, G. T. (2015). Robust aerodynamic design optimization using polynomial chaos. Journal of Aircraft, 46(2):635-646.

Feng, L., Ze-Yu, M., Zheng-Fei, T., and Yong-Fu, C. (2013). Uncertainty analysis of vehicle suspension systems based on polynomial chaos methods. In Proceedings - 2013 4th International Conference on Intelligent Systems Design and Engineering Applications, ISDEA 2013, pages 732-735.

Isukapalli, S. S. (1999). Uncertainty Analysis of Transport-Transformation Models. PhD thesis, The State University of New Jersey.

Kewlani, G., Crawford, J., and lagnemma, K. (2012). A polynomial chaos approach to the analysis of vehicle dynamics under uncertainty. Vehicle System Dynamics, 50:1-26.

Le, T. D. and Ahn, K. K. (2013). Experimental investigation of a vibration isolation system using negative stiffness structure. International Journal of Mechanical Sciences, 70(5):99-112.

Li, W., Lu, Z., and Zhang, D. (2009). Stochastic analysis of unsaturated flow with probabilistic collocation method. Water Resources Research, 45(8).

Liu, H. (2021). DATA FOR "Uncertainty Analysis of Quasi-Zero Stiffness Air Suspension based on Polynomial Chaos Method". https://doi.org/10.7910/DVN/YOORSR.

Nagy, Z. and Braatz, R. (2010). Distributional uncertainty analysis using polynomial chaos expansions. In Proceedings of the IEEE International Symposium on Computer-Aided Control System Design, pages 1103 1108.

Palomares, E., Nieto, A. J., Morales, A. L., Chicharro, J. M., and Pintado, P. (2018). Numerical and experimental analysis of a vibration isolator equipped with a negative stiffness system. Journal of Sound and Vibration, 414:31-42.

Qiu, C., Peng, X., Liu, Z., and Tan, J. (2019). Sensitivity analysis of random and interval uncertain variables based on polynomial chaos expansion method. IEEE Access, PP(99):1-1.

Sandu, A., Sandu, C., and Ahmadian, M. (2006). Modeling multibody systems with uncertainties. part i: Theoretical and computational aspects. Multibody System Dynamics, 15(4):369-391.

Wiener, N. (1938). The homogeneous chaos. American Journal of Mathematics, 60(4):897-936.

Xiu, D. (2007). Efficient collocational approach for parametric uncertainty analysis. Communications in Computational Physics, 2(2):293-309.

Xiu, D. and Karniadakis, G. (2002). The wiener-askey polynomial chaos for stochastic differential equations. SIAM J. Sci. Comput., 24:619-644.

Xiu, D. and Karniadakis, G. E. (2003a). Modeling uncertainty in flow simulations via generalized polynomial chaos. Journal of Computational Physics, 187(1):137-167.

Xiu, D. and Karniadakis, G. E. (2003b). A new stochastic approach to transient heat conduction modeling with uncertainty. International Journal of Heat and Mass Transfer, 46(24):4681-4693.

Figures and Tables 


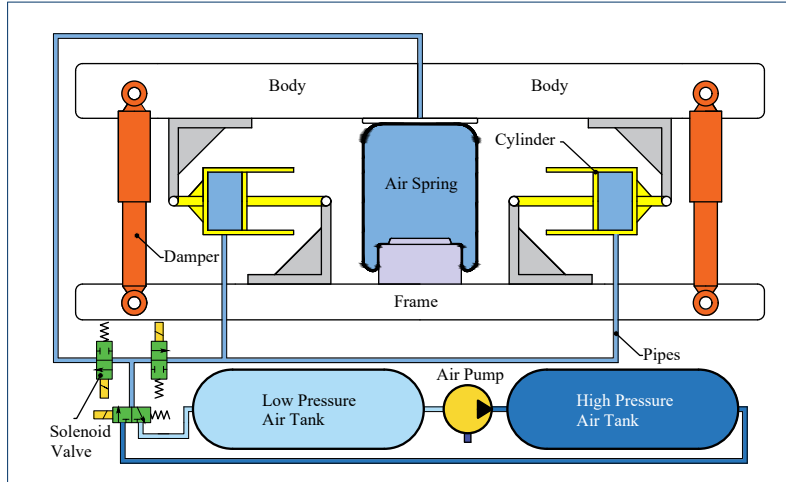

Figure 1 The structure of quasi-zero stiffness air suspension.

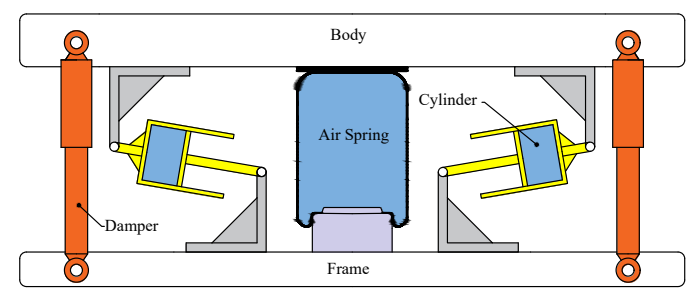

(a) Movement of the suspension when the distance increases.

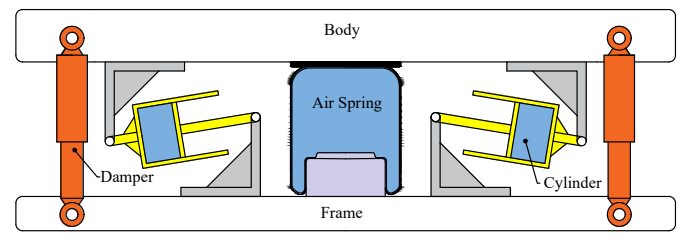

(b) Movement of the suspension when the distance decreases.

Figure 2 Suspension movement when the distance between the body and the frame changes.

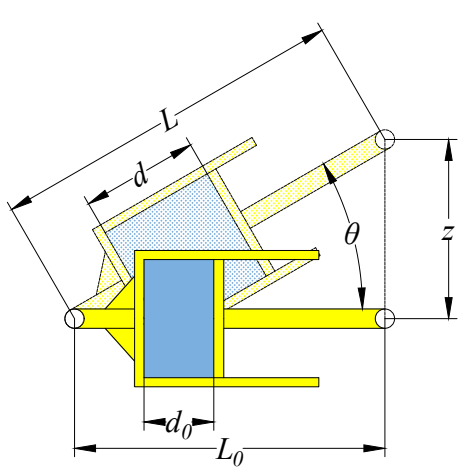

Figure 3 Geometric relationship of cylinder motion.
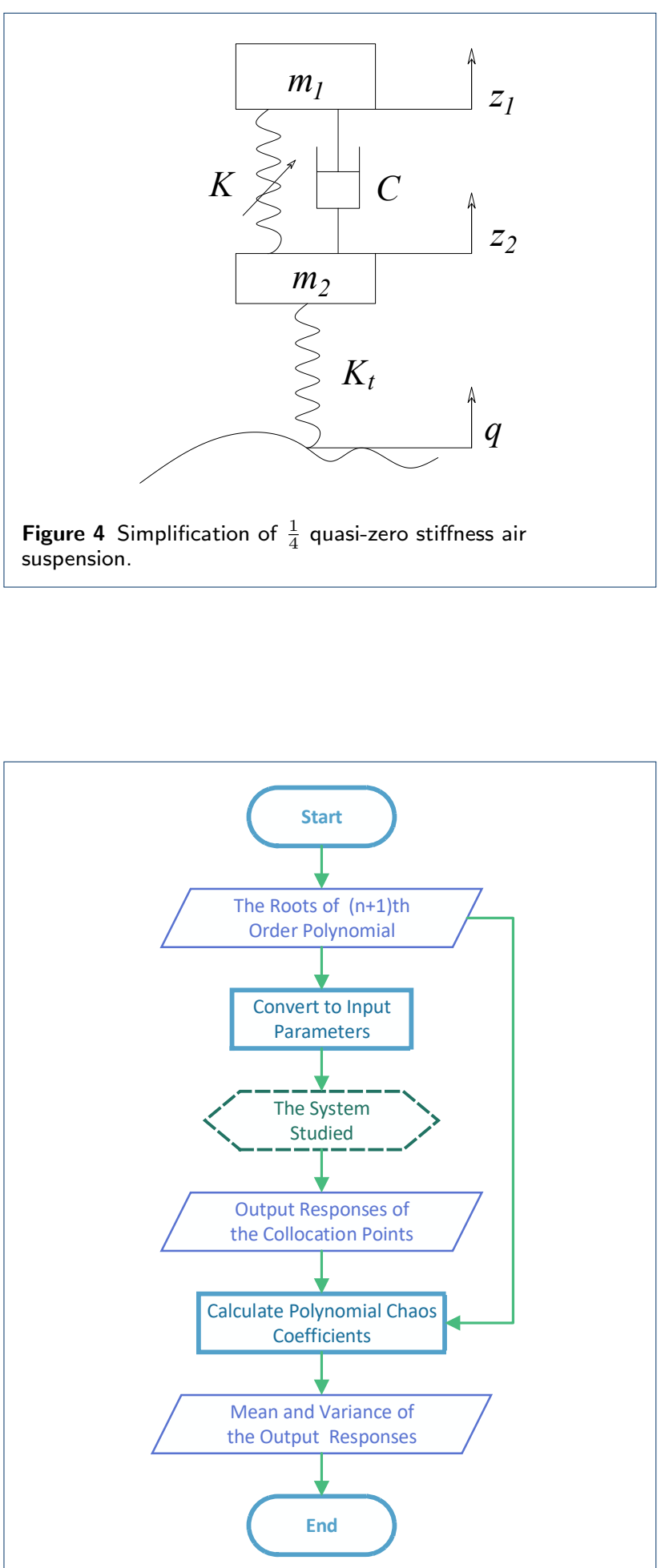

Figure 5 Process for calculating output response statistics using the PCE method. 


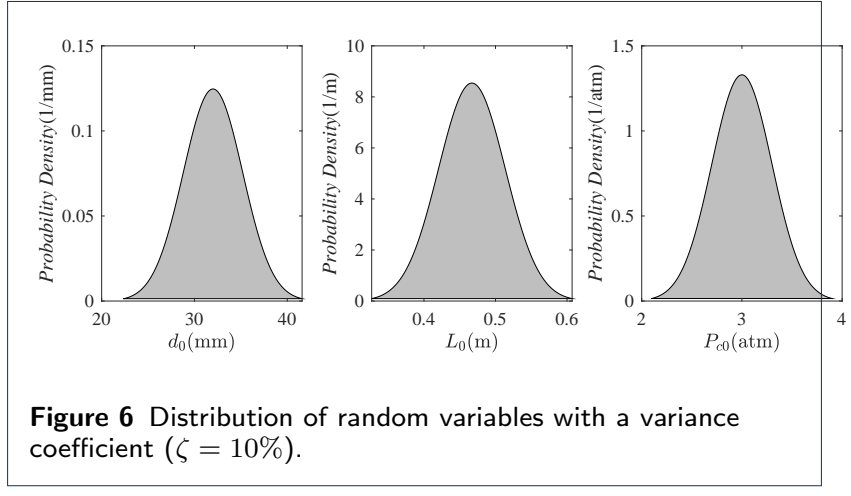

Table 1 The normalized Hermite polynomials.

\begin{tabular}{cc}
\hline Polynomial Order & Hermite polynomials \\
\hline 0th & 1 \\
1 th & $\xi$ \\
2 th & $\frac{1}{\sqrt{2}}\left(\xi^{2}-1\right)$ \\
3 th & $\frac{1}{\sqrt{6}}\left(\xi^{3}-3 \xi\right)$ \\
4 th & $\frac{1}{2 \sqrt{6}}\left(\xi^{4}-6 \xi^{2}+3\right)$ \\
5 th & $\frac{1}{2 \sqrt{30}}\left(\xi^{5}-10 \xi^{3}+15 \xi\right)$ \\
\hline
\end{tabular}

Table 2 The parameters of QZSAS.

\begin{tabular}{cc}
\hline Parameters & Value \\
\hline Air polytropic index, $n_{s}, n_{c}$ & 1 \\
Cylinder node distance, $L_{0}$ & $0.467 \mathrm{~m}$ \\
Distance between cylinder piston & $32 \mathrm{~mm}$ \\
and end face, $d_{0}$ & $0.0314 \mathrm{~m}^{2}$ \\
Cylinder piston area, $A_{c}$ & $0.0421 \mathrm{~m}^{2}$ \\
Effective area of air spring, $A_{s}$ & $2000 \mathrm{Ns} / \mathrm{m}$ \\
Shock absorber damping factor, $C$ & $100 \mathrm{~kg}$ \\
Wheel mass, $m_{2}$ & $800 \mathrm{~kg}$ \\
body mass, $m_{1}$ & $243000 \mathrm{~N} / \mathrm{m}$ \\
Tire Stiffness, $K_{t}$ & $0.275 \mathrm{~m}$ \\
Initial height of air spring, $z_{0}$ & $2.88 \mathrm{~atm}$ \\
Air spring initial air pressure, $P_{s 0}$ & $3 \mathrm{~atm}$ \\
Cylinder initial air pressure, $P_{c 0}$ &
\end{tabular}

Table 3 Body acceleration $\mathrm{RMM}\left(\mathrm{m} / \mathrm{s}^{2}\right), \mathrm{RVM}\left(\mathrm{m}^{2} / \mathrm{s}^{4}\right)$ induced by random variable inputs with different coefficients of variation.

\begin{tabular}{ccccc}
\hline \multirow{2}{*}{$\begin{array}{l}\text { Input Random } \\
\text { Variables }\end{array}$} & \multicolumn{2}{c}{$\zeta=10 \%$} & \multicolumn{2}{c}{$\zeta=20 \%$} \\
\cline { 2 - 5 } & RMM & RVM & RMM & RVM \\
\hline$d_{0}$ & 23.4278 & 0.6113 & 22.7419 & 2.3366 \\
$L_{0}$ & 23.4799 & 0.3698 & 22.9516 & 1.4444 \\
$P_{c 0}$ & 23.0401 & 1.9468 & 21.3869 & 7.5703 \\
\hline
\end{tabular}

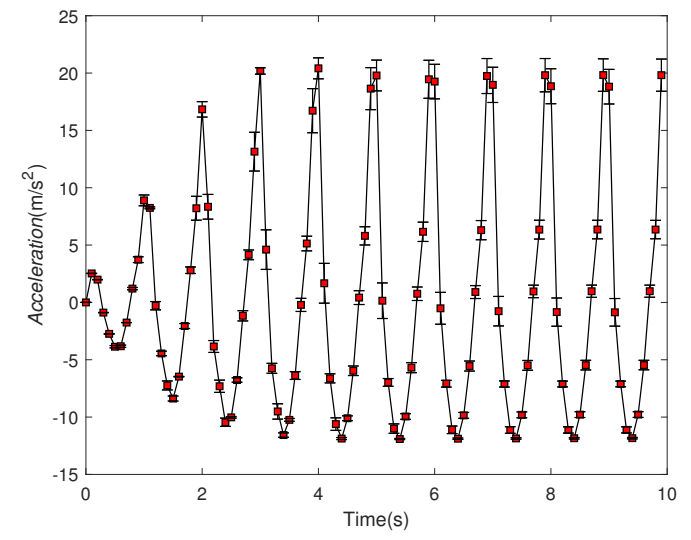

(a) Effect of $d_{0}$ on body acceleration.

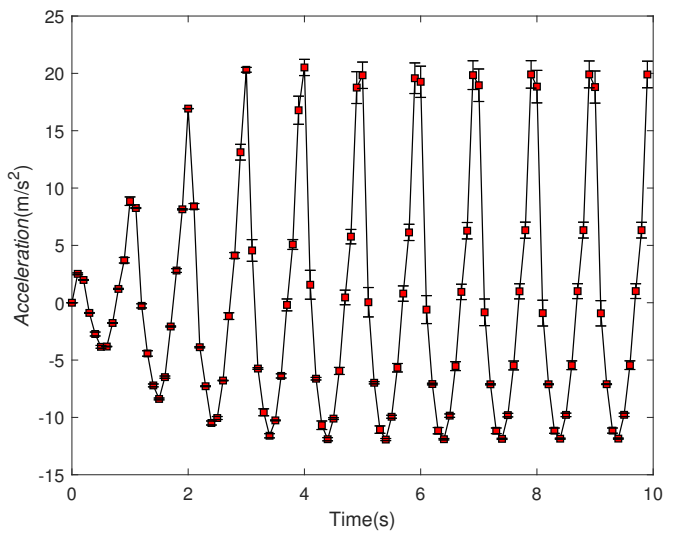

(b) Effect of $L_{0}$ on body acceleration.

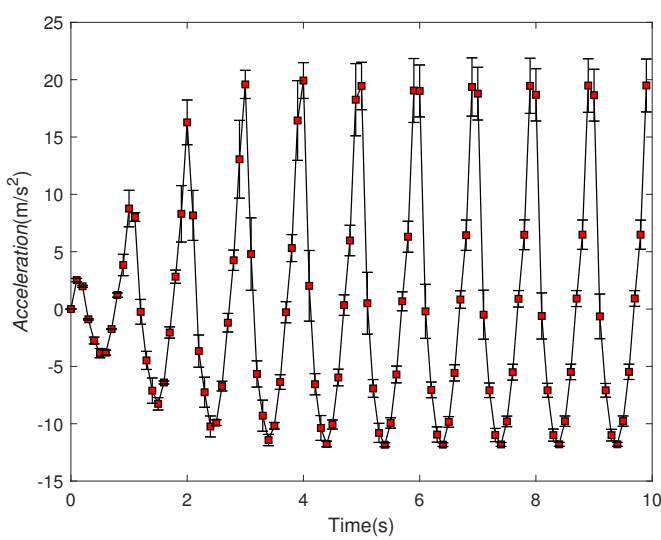

(c) Effect of $P_{c 0}$ on body acceleration.

Figure 7 The effect of different parameter uncertainties on the mean and standard deviation of body acceleration. 


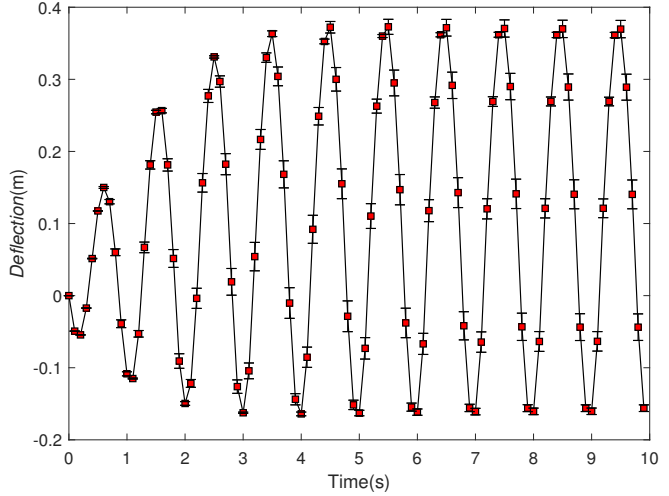

(a) Effect of $d_{0}$ on dynamic deflection.

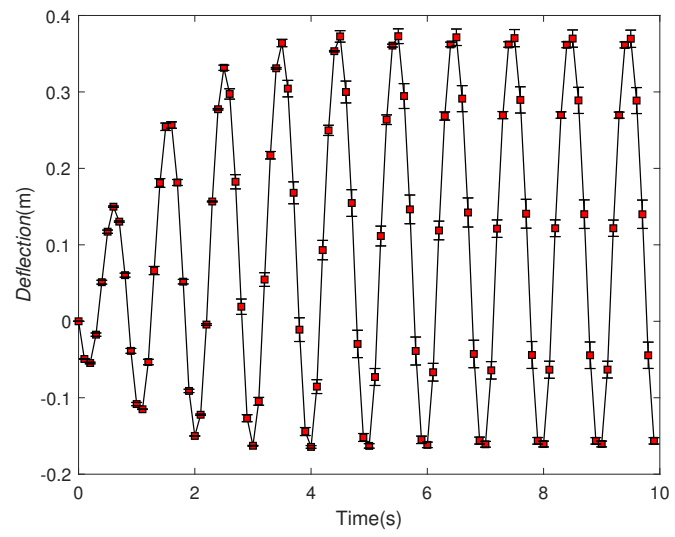

(b) Effect of $L_{0}$ on dynamic deflection.

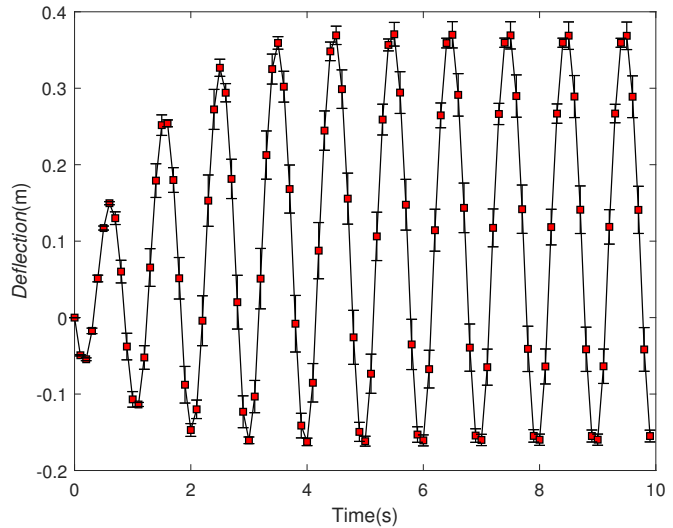

(c) Effect of $P_{c 0}$ on dynamic deflection.

Figure 8 The effect of different parameter uncertainties on the mean and standard deviation of dynamic deflection.

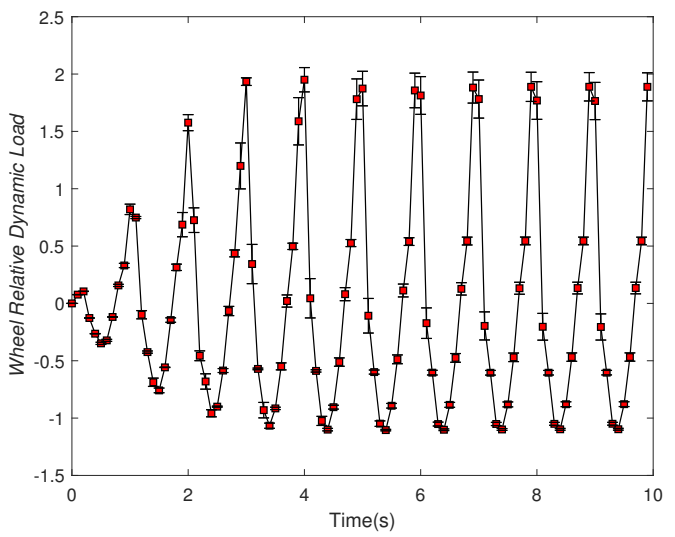

(a) Effect of $d_{0}$ on dynamic load.

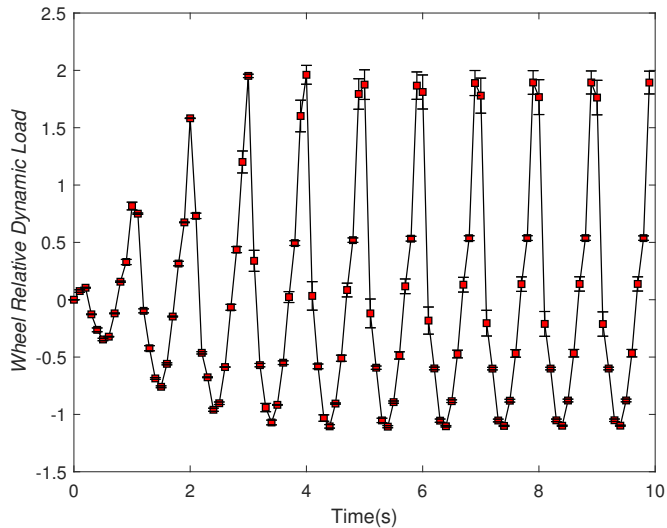

(b) Effect of $L_{0}$ on dynamic load.

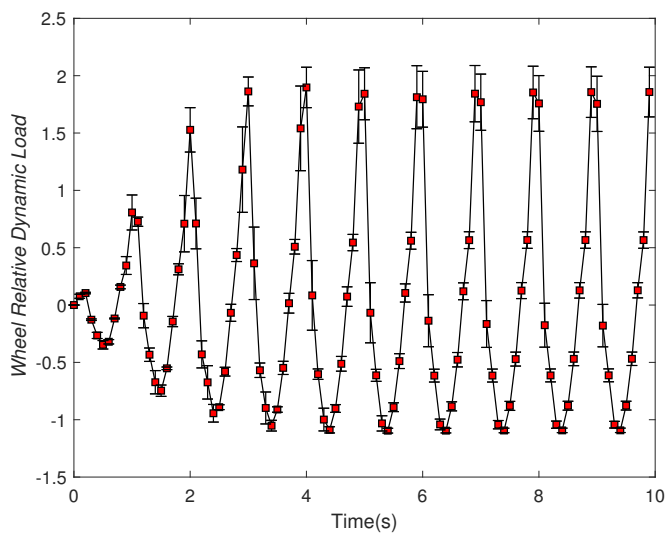

(c) Effect of $P_{c 0}$ on dynamic load.

Figure 9 The effect of different parameter uncertainties on the mean and standard deviation of dynamic load. 


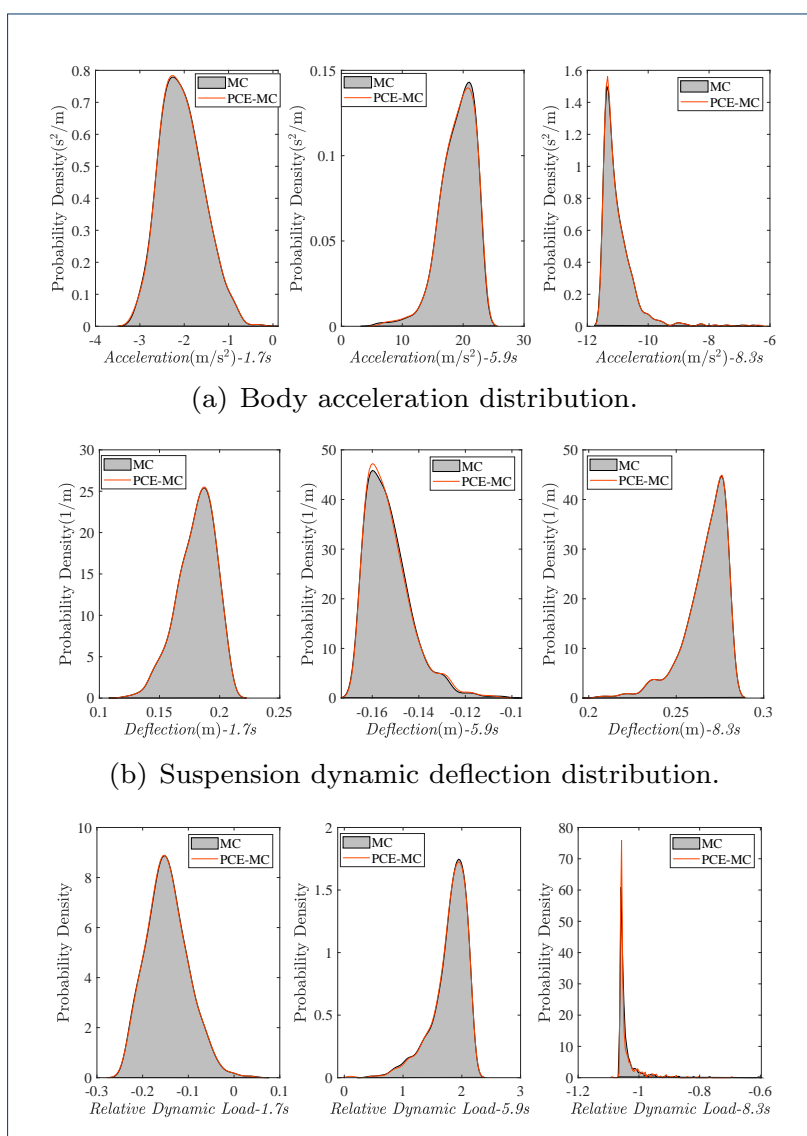

(c) Wheel ralative dynamic load distribution.

Figure 10 Distribution of output random variables.

Table 4 Dynamic deflection of suspension $\operatorname{RMM}(\mathrm{m}), \mathrm{RVM}\left(\mathrm{m}^{2}\right)$ induced by random variable inputs with different coefficients of variation

\begin{tabular}{ccccc}
\hline \multirow{2}{*}{$\begin{array}{c}\text { Input Random } \\
\text { Variables }\end{array}$} & \multicolumn{2}{c}{$\zeta=10 \%$} & \multicolumn{2}{c}{$\zeta=20 \%$} \\
\cline { 2 - 5 } & RMM & RVM & RMM & RVM \\
\hline$d_{0}$ & 0.3784 & 0.0001 & 0.3740 & 0.0006 \\
$L_{0}$ & 0.3785 & 0.0001 & 0.3748 & 0.0004 \\
$P_{c 0}$ & 0.3760 & 0.0004 & 0.3618 & 0.0021 \\
\hline
\end{tabular}

Table 5 Wheel ralative dynamic load RMM,RVM induced by random variable inputs with different coefficients of variation

\begin{tabular}{ccccc}
\hline \multirow{2}{*}{$\begin{array}{c}\text { Input Random } \\
\text { Variables }\end{array}$} & \multicolumn{2}{c}{$\zeta=10 \%$} & \multicolumn{2}{c}{$\zeta=20 \%$} \\
\cline { 2 - 5 } & $\mathrm{RMM}$ & $\mathrm{RVM}$ & $\mathrm{RMM}$ & $\mathrm{RVM}$ \\
\hline$d_{0}$ & 2.2355 & 0.0060 & 2.1707 & 0.0228 \\
$L_{0}$ & 2.2436 & 0.0037 & 2.1948 & 0.0144 \\
$P_{c 0}$ & 2.1957 & 0.0190 & 2.0164 & 0.0701 \\
\hline
\end{tabular}

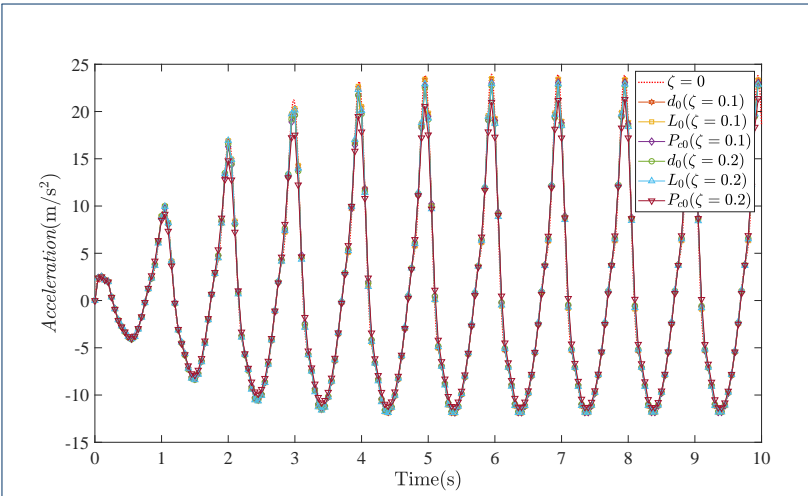

(a) Body acceleration response.

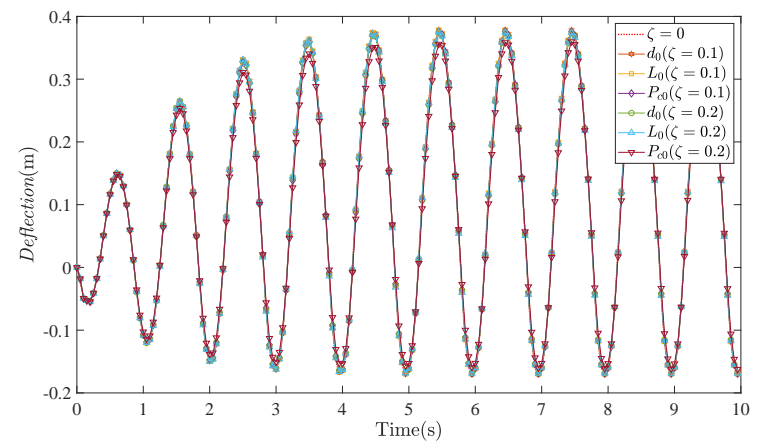

(b) Suspension dynamic deflection response.

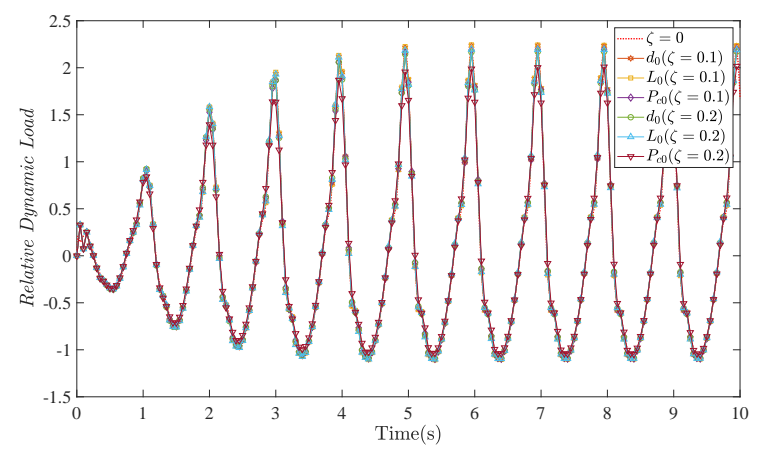

(c) Wheel ralative dynamic load response.

Figure 11 Effect of random variables with different coefficients of variation on the mean values of the responses under sinusoidal road excitation input. 


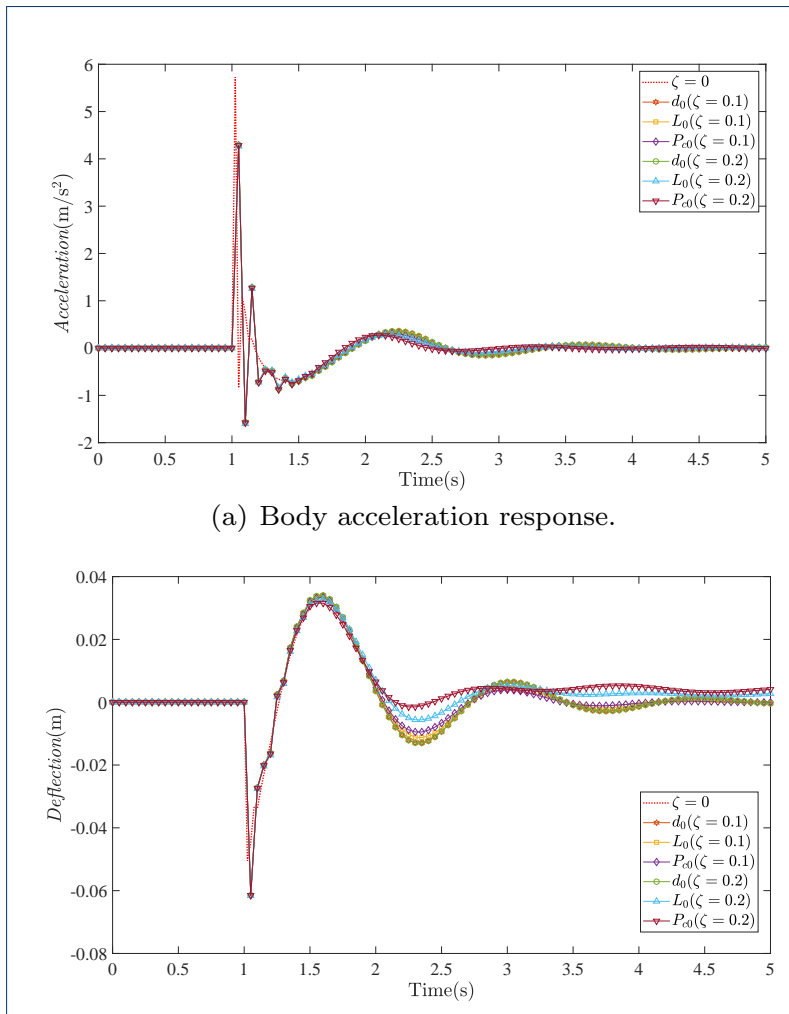

(b) Suspension dynamic deflection response.

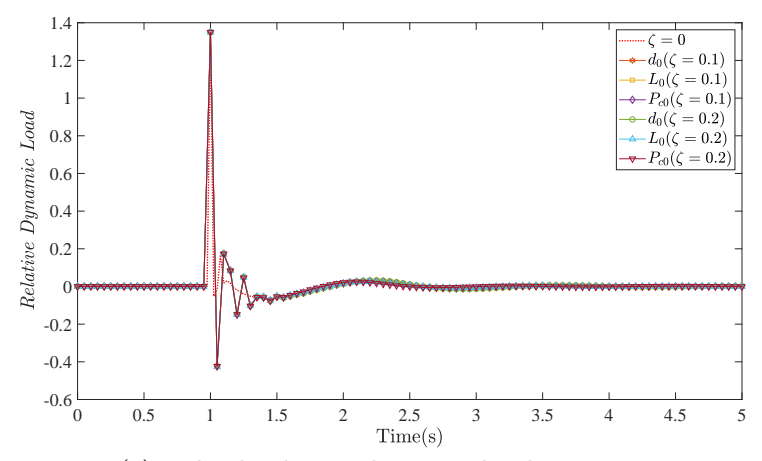

(c) Wheel ralative dynamic load response.

Figure 12 Effect of random variables with different coefficients of variation on the mean values of the responses under step road excitation input.

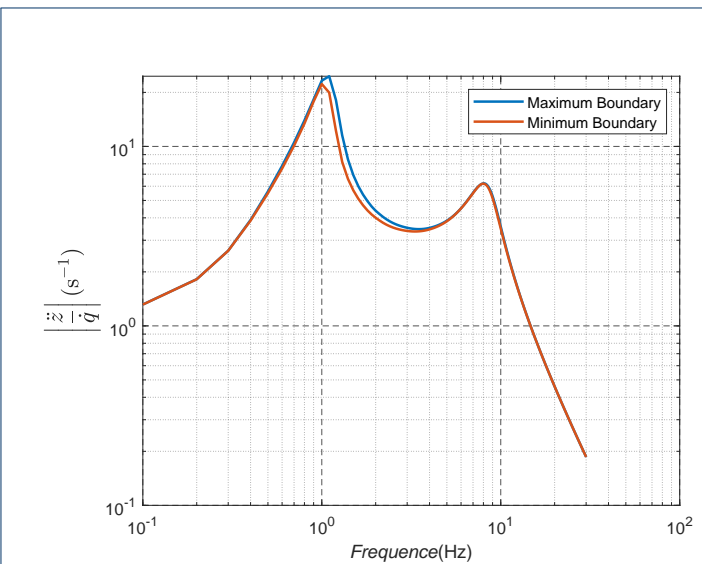

(a) Body acceleration response.

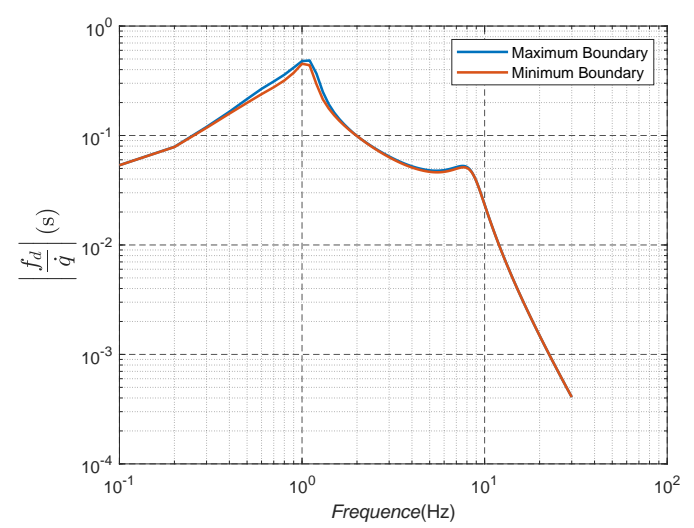

(b) Suspension dynamic deflection response.

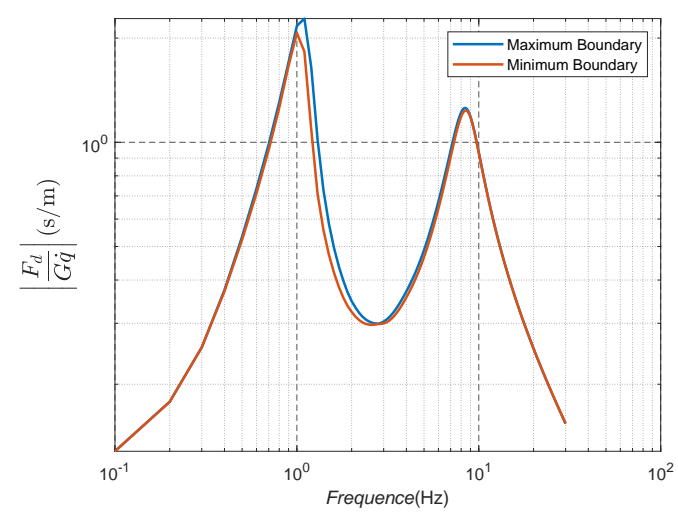

(c) Wheel ralative dynamic load response.

Figure 13 Effect of $d_{0}$ uncertainty on $a, f_{d}$, and $F_{d} / G$ vs. $\dot{q}$ amplitude-frequency characteristics. 


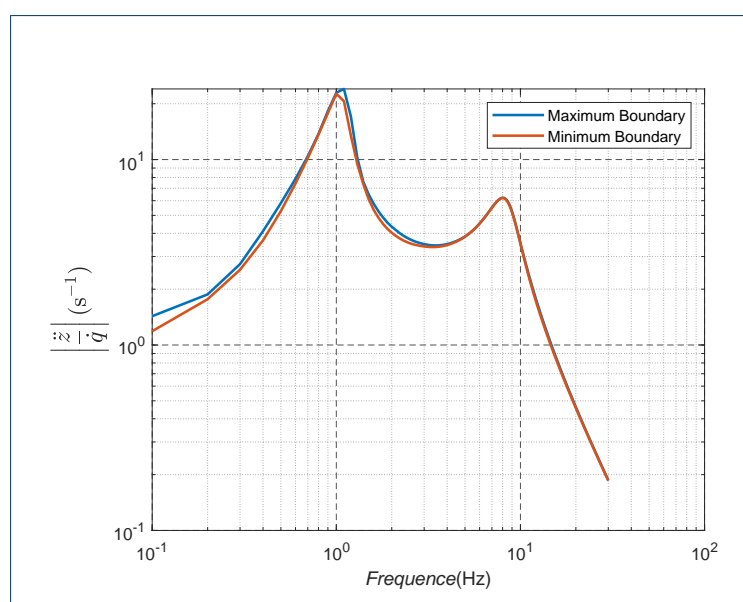

(a) Body acceleration response.

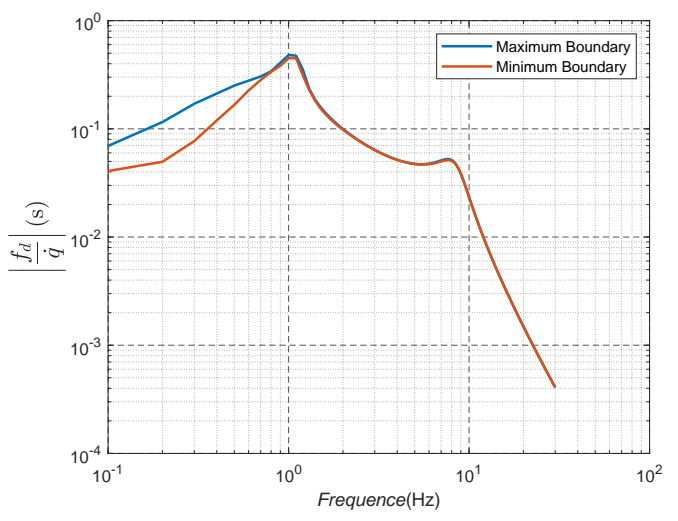

(b) Suspension dynamic deflection response.

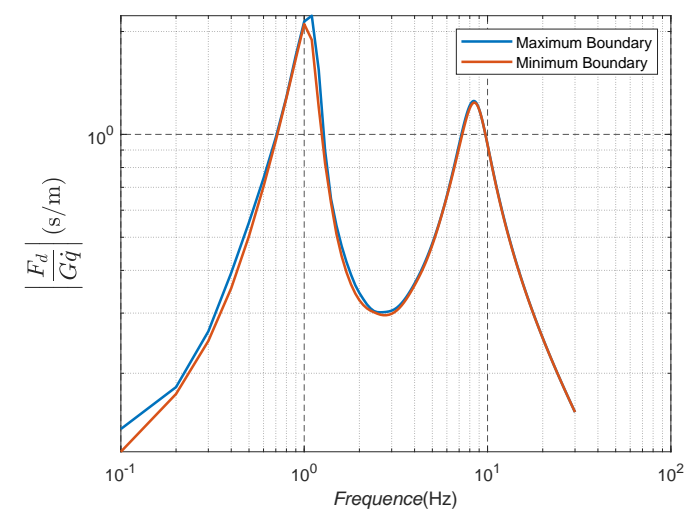

(c) Wheel ralative dynamic load response.

Figure 14 Effect of $L_{0}$ uncertainty on $a, f_{d}$, and $F_{d} / G$ vs. $\dot{q}$ amplitude-frequency characteristics.

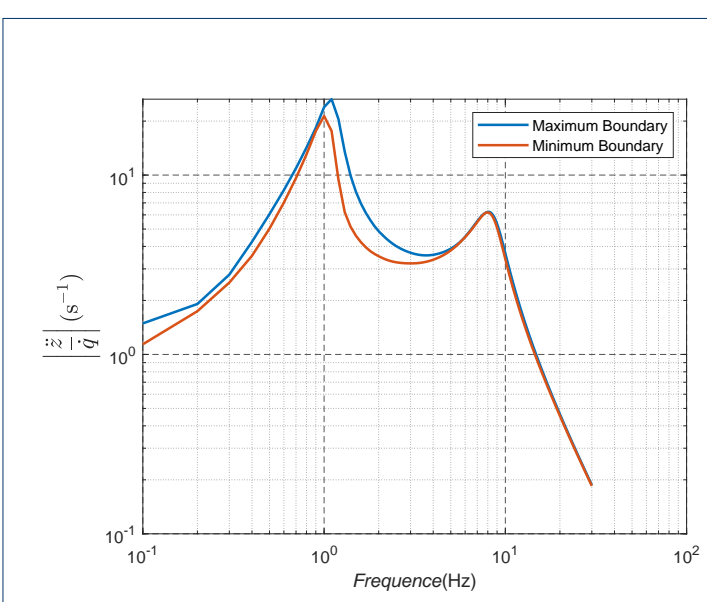

(a) Body acceleration response.

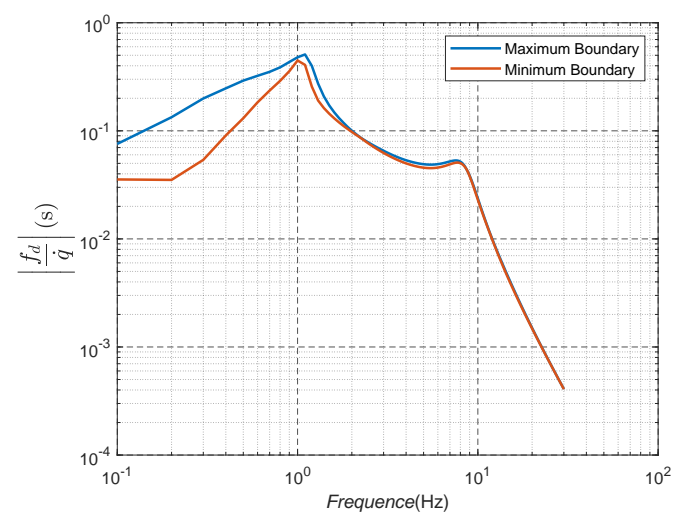

(b) Suspension dynamic deflection response.

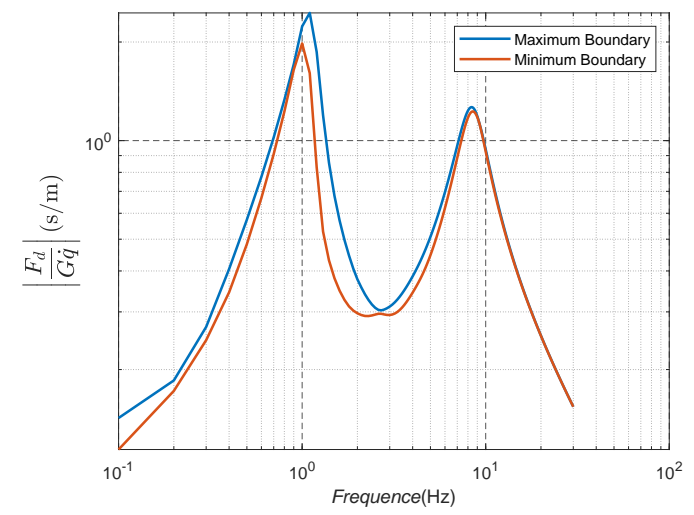

(c) Wheel ralative dynamic load response.

Figure 15 Effect of $P_{c 0}$ uncertainty on $a, f_{d}$, and $F_{d} / G$ vs. $\dot{q}$ amplitude-frequency characteristics. 